\title{
ON CARTAN PSEUDO GROUPS
}

\author{
A. M. RODRIGUES
}

Let $M$ be a domain in an Euclidean space and let $\Gamma$ be a pseudo group of transformations* of $M$. We say that $\Gamma$ is a Cartan pseudo group $[1,2]$ if the following conditions are satisfied:

1) There exists a domain $M^{\prime}$ and a projection $\rho: M \rightarrow M^{\prime}$, such that the orbits of $\Gamma$ are the fibers of the projection $\rho$. We assume moreover that there is a system of coordinates $(x)$ of $M^{\prime}$ and a system of coordinates $(x, y)$ of $M$ such that the fibers of $\rho$ are defined by $(x)=$ constants,

2) There are forms $\omega^{i}, \widetilde{\omega}^{\lambda}, i=1 \cdots m, \lambda=1 \cdots n$, defined in $D$ such that

a) $\left(\omega^{i}, \widetilde{\omega}^{\lambda}\right)$ is a basis of the space of linear forms at every point of $M$,

$$
\text { b) } d \omega^{i}=\frac{1}{2} c_{j k \omega^{j}}^{i} \wedge \omega^{k}+a_{j_{\lambda}}^{i} \omega^{j} \wedge \widetilde{\omega}^{\lambda}
$$

where $c_{j k}^{i}, a_{j \lambda}^{i}$ are functions on $M$ which depend on $(x)$ only,

c) $\omega^{r}=d x^{r}$ for $1 \leq r \leq$ dimension $M^{\prime}$,

d) The matrices $a_{\lambda}=\left\|a_{j \lambda}^{i}\right\|$ are linearly independent at every point of $M$,

e) Let $\pi_{1}$ and $\pi_{2}$ be respectively the projections of $M \times M$ into the first and second factors. The closed differential system $\Sigma$ on $M \times M$, with independent variables $x \circ \pi_{1}, y \circ \pi_{1}$ generated by

$$
\begin{array}{ll}
x^{r} \circ \pi_{1}-x^{r} \circ \pi_{2}=0, & 1 \leq r \leq \text { dimension } M^{\prime}, \\
\pi_{1}^{*} \omega^{i}-\pi_{2}^{*} \omega^{i}=0 & 1 \leq i \leq m
\end{array}
$$

is in involution at every integral point,

3) A local transformation $f$ of $M$ is in $\Gamma$ if and only if $f$ preserves the forms $\omega^{i}$, i.e. $f^{*} \omega^{i}=\omega^{i}, i=1, \ldots, m$.

In this note we prove that every differential form on $M$ which is invariant under all transformations of a Cartan pseudo group $\Gamma$ is a linear combination of the forms $\omega^{i}$ the coefficient being functions of $x$ only.

Received on 1 May, 1962.

* All maps and differential forms considered in this note are assumed to be analytic. 
Put $\sigma^{i}=\pi_{1}^{*} \omega^{i}-\pi_{2}^{*} \omega^{i}, \tau^{\lambda}=\pi_{1}^{*} \widetilde{\omega}^{\lambda}-\pi_{2}^{*} \widetilde{\omega}^{\lambda}$. The set $\left(\pi_{1}^{*} \omega^{i}, \pi_{1}^{*} \widetilde{\omega}^{\lambda}, \sigma^{i}, \tau^{\lambda}\right)$ is a basis of differential forms on every point of $M \times M$. Let $E$ be a contact element of $M \times M$ at the integral point $\left(u_{1}, u_{2}\right) \in M \times M$ such that the forms $\pi_{1}^{*} \omega^{i}$ and $\pi_{1}^{*} \widetilde{\omega}^{\lambda}$ are linearly independent on $E$ and denote by $\sigma^{i} / E$ the restriction of $\sigma^{i}$ to $E$. Put $x=\rho\left(u_{1}\right)=\rho\left(u_{2}\right)$ and write

$$
\begin{aligned}
& \sigma^{i} / E=p_{j}^{i}\left(\pi_{1}^{*} \omega^{j}\right) / E+p_{\lambda}^{i}\left(\pi_{1}^{*} \widetilde{\omega}^{\lambda}\right) / E \\
& \tau^{\lambda} / E=q_{j}^{\lambda}\left(\pi_{1}^{*} \omega^{j}\right) / E+q_{\mu}^{\lambda}\left(\pi_{1}^{*} \widetilde{\omega}^{\mu}\right) / E .
\end{aligned}
$$

$E$ is an integral element of $\Sigma$ if and only if

$$
\begin{aligned}
& p_{j}^{i}=p_{\lambda}^{i}=q_{\mu}^{\lambda}=0 \\
& a_{j \lambda}^{i}(x) q_{k}^{\lambda}-a_{k_{\lambda}}^{i}(x) q_{j}^{\lambda}=0
\end{aligned}
$$

for every choice of the indices $i, j, k$.

Let $V$ and $V^{\prime}$ be vector spaces of dimensions $v$ and $v^{\prime}$ over the real field and let $L$ be a vector space of linear maps of $V$ into $V^{\prime}$ of dimension $n$. Take basis of $V$ and $V^{\prime}$ and let $a_{\lambda}=\left\|a_{j_{\lambda}}^{i}\right\|, i=1, \ldots, v^{\prime}, j=1, \ldots, v, \lambda=1, \ldots, n$ be a basis of $L$. The space $\mathscr{D}(L)$ of all linear maps $b: V \rightarrow L, b=\left\|b_{j}^{\lambda}\right\|$ such that

$$
a_{j \lambda}^{i} b_{k}^{\lambda}-a_{k \lambda}^{i} b_{j}^{\lambda}=0
$$

for every choice of $i, j, k$, is called the derived space of $L$.

Let $s_{1}$ be the maximum rank of the matrix $A_{1}=\left\|a_{j \lambda}^{i} t_{1}^{j}\right\|$ when the vector $\left(t_{1}^{1}, t_{1}^{2}, \ldots, t_{1}^{v}\right)$ varies in $R^{v}$. Put $A_{2}=\left\|a_{j \lambda}^{i} t_{2 \|}^{j}\right\|$ and let $s_{2}$ be the maximum rank of the matrix $\left\|\begin{array}{l}A_{1} \\ A_{2}\end{array}\right\|$ when the vectors $\left(t_{1}^{1}, \ldots, t_{1}^{v}\right),\left(t_{2}^{1} \cdots t_{2}^{v}\right)$, vary independently in $R^{v}$. Define an integer $s_{i}$ in a similar way for each $i, 1 \leq i \leq v-1$. The integers $s_{i}$ are called the characters of $L$. If $\delta$ is the dimension of $\mathscr{D}(L)$ it can be proved $[3$, page 4$]$ that

$$
\delta \leq n \cdot v-\left(s_{1}+\cdots+s_{v-1}\right) .
$$

The space $L$ is called involutive when the equality holds in (3).

Let $L(x)$ be the space of endomorphisms of $R^{m}$ generated by the matrices $a_{\lambda}(x)=\left\|a_{j \lambda}^{i}(x)\right\|$ and denote by $s_{1}(x), \ldots, s_{m-1}(x), \delta(x)$ the characters and the dimension of the derived space of $L(x) ; \Sigma$ is in involutions at every integral point if and only if $\delta(x)$ is constant and $L(x)$ is involutive for every $x$. When $\Sigma$ is involutive the characters $s_{i}(x)$ are independent of $x$. 
Let now $f$ be a transformation of $\Gamma$. Applying $f^{*}$ to equation (1) we have

$$
d \omega^{i}=\frac{1}{2} c_{j k}^{i} \omega^{j} \wedge \omega^{k}+a_{j k}^{i} \omega^{j} \wedge f^{*} \widetilde{\omega}^{\lambda}
$$

hence,

$$
a_{j_{\lambda}}^{i}\left(f^{*} \widetilde{\omega}^{\lambda}-\widetilde{\omega}^{\lambda}\right) \wedge \omega^{j}=0
$$

and the linear form $a_{j \lambda}^{i}\left(f^{*} \widetilde{\omega}^{\lambda}-\widetilde{\omega}^{\lambda}\right)$ is a linear combination of the form $\omega^{j}$. Since the matrices $a_{\lambda}$ are linearly independent we have

$$
f^{*} \widetilde{\omega}^{\lambda}=\widetilde{\omega}^{\lambda}+h_{j}^{\lambda} \omega^{j} .
$$

Substituting (4) in (1) we have

$$
a_{j \lambda}^{i} h_{k}^{\lambda}-a_{j \lambda}^{i} h_{j}^{\lambda}=0 .
$$

Conversely let $u_{1}, u_{2}$ be two points of $M$ such that $\rho\left(u_{1}\right)=\rho\left(u_{2}\right)=x$ and let $h_{j}^{\lambda}$ be an element of the derived space of $L(x)$. Let $E$ be the integral contact element of $\Sigma$ at the point $\left(u_{1}, u_{2}\right) \in M \times M$ whose coordinates are $p_{j}^{i}=p_{\lambda}^{i}=q_{\mu}^{\lambda}=0$, $q_{j}^{\lambda}=h_{j}^{\lambda}$. Let $f$ be the transformation of $M$ defined by an integral manifold of $\Sigma$ whose tangent space at the point $\left(u_{1}, u_{2}\right)$ is $E$. Then $f\left(u_{1}\right)=u_{2}$ and, at the point $\boldsymbol{u}_{1}$

$$
f^{*} \widetilde{\omega}^{\lambda}=\widetilde{\omega}^{\lambda}+h_{i}^{\lambda} \omega^{i}
$$

for $1 \leq \lambda \leq n$.

Assume now that the differential form $\omega$ is invariant under $\Gamma$ and write

$$
\omega=\alpha_{i} \omega^{i}+\beta_{\lambda} \widetilde{\omega}^{\lambda} \text {. }
$$

Given $u_{1}, u_{2} \in M$ with $\rho\left(u_{1}\right)=\rho\left(u_{2}\right)=x$ there exists $f \in \Gamma$ such that $f\left(u_{1}\right)=u_{2}$ and, at the point $u_{1}, f^{*} \widetilde{\omega}^{\lambda}=\widetilde{\omega}^{\lambda}$. It follows that $\alpha_{i}, \beta_{\lambda}$ depend only on $x$. Assume that there exists $x$ such that not all coefficients $\beta_{\lambda}(x)$ are zero. Then we can take $\omega$ to be one of the forms $\widetilde{\omega}^{\lambda}$. Hence, there exists a system of forms $\left(\omega^{i}, \widetilde{\omega}^{\lambda}\right)$ which satisfy conditions 2) and 3) and such that $\widetilde{\omega}^{n}$ is an invariant form of $\Gamma$. Then, if $h_{i}^{\lambda}$ is an element of $\mathscr{D}(L(x))$ we have necessarily $h_{i}^{n}=0$ for every $i$. Let $L_{0}$ be the subspace $L(x)$ generated by $a_{1}(x), \ldots, a_{n-1}(x)$. Any element of $\mathscr{D}(L(x))$ has values in $L_{0}$ hence, the dimension of $\mathscr{D}\left(L_{0}\right)$ is $\delta(x)$. Let $s_{1}^{\prime} \cdots s_{m-1}^{\prime}$ be the characters of $\left(L_{0}\right)$. By (3)

$$
\delta(x) \leq m(n-1)-\left[s_{1}^{\prime}+\cdots+s_{m-1}^{\prime}\right] .
$$

By the definition of the characters $s_{i} \leq s_{i}^{\prime}+1$. Therefore 


$$
\delta(x)<m \cdot n-\left[s_{1}+\cdots+s_{m-1}\right]
$$

and $L(x)$ is not involutive. Hence all coefficients $\beta$ are zero and $\omega$ is a linear combination of the $\omega^{i}$ with coefficients depending on $x$ only.

The following example shows that the result is not true if we drop the condition that $\Sigma$ is in involution even if the coefficients $a_{j \lambda}^{i}, c_{j k}^{i}$ are constant and $\Gamma$ is transitive. Let $\Gamma$ be the pseudo group operating on $R^{n}$ obtained by localization of the group of rigid motions. $\Gamma$ is a Lie pseudo group of order 1. Let $\mathscr{F}$ be the space of orthonormal frames of $R^{n}$ and denote by $\tilde{I}$ the prolongation of $\Gamma$ to $\mathscr{H}$. In $\mathscr{H}$ we have differential forms $\omega^{i}, \omega_{j}^{i}\left(\omega_{j}^{i}+\omega_{i}^{j}=0\right)$, canonically defined which satisfy equations (1) with constant coefficients. A transformation $f$ of $\mathscr{H}$ is in $\tilde{T}$ if and only if $f$ preserves the forms $\omega^{i}$. On the other hand all the forms $\omega^{i}, \omega_{j}^{i}$ are invariant by the elements of $\hat{\Gamma}$.

\section{References}

[1] E. Cartan, Sur la structure des groupes infinis de transformations; Ann. Ec. Normale, t. 21, 1904.

[2] M. Kuranishi, On the local theory of continuous infinite pseudo groups II. Nagoya Mathematical Journal, 1961.

[3] Y. Matsushima, Sur les algèbres de Lie linèaires semi-involutives, Colloque de Topologie de Strasbourg, 1954.

\section{Princeton University}

